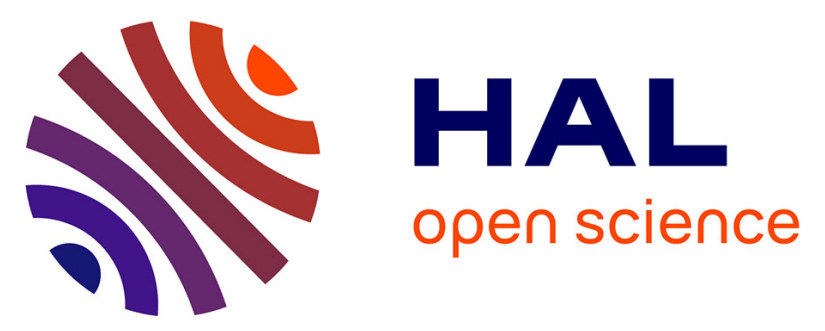

\title{
Stratégies de subsistance et perception des aliments des populations autochtones de Yakoutie Arctique (Districts de Basse et de Moyenne Kolyma)
}

C. Malet, Boris Chichlo, Joëlle Robert-Lamblin, Claude Marcel Hladik, Patrick Pasquet

\section{- To cite this version:}

C. Malet, Boris Chichlo, Joëlle Robert-Lamblin, Claude Marcel Hladik, Patrick Pasquet. Stratégies de subsistance et perception des aliments des populations autochtones de Yakoutie Arctique (Districts de Basse et de Moyenne Kolyma). Bulletins et Mémoires de la Société d'anthropologie de Paris, 2003, 15 (1-2), pp.101-120. hal-00276533

\section{HAL Id: hal-00276533 \\ https://hal.science/hal-00276533}

Submitted on 15 Feb 2013

HAL is a multi-disciplinary open access archive for the deposit and dissemination of scientific research documents, whether they are published or not. The documents may come from teaching and research institutions in France or abroad, or from public or private research centers.
L'archive ouverte pluridisciplinaire HAL, est destinée au dépôt et à la diffusion de documents scientifiques de niveau recherche, publiés ou non, émanant des établissements d'enseignement et de recherche français ou étrangers, des laboratoires publics ou privés. 


\section{STRATÉGIES DE SUBSISTANCE ET PERCEPTION DES ALIMENTS DES POPULATIONS AUTOCHTONES DE YAKOUTIE ARCTIQUE (DISTRICTS DE BASSE ET DE MOYENNE KOLYMA)}

\section{SUBSISTENCE PATTERNS, FOOD PERCEPTION AND PREFERENCES AMONG INDIGENOUS PEOPLE OF ARCTIC YAKUTIA (LOWER AND MIDDLE KOLYMA DISTRICTS)}

Christain MALET ${ }^{1}$, Boris ChICHLO ${ }^{1}$, Joëlle ROBERT-LAMBLIN ${ }^{1}$, Claude-Marcel HLADIK ${ }^{2}$, Patrick PASQUET ${ }^{2}$

RÉSUMÉ

Une étude des appréciations de différents aliments et de la perception gustative a été réalisée sur un échantillon de populations autochtones de Yakoutie arctique, composé de 102 Evènes, 52 Yakoutes et 22 Tchouktches. Afin de compléter les réponses, sur des échelles analogiques, correspondant à 69 items alimentaires, des tests en simple aveugle avec des solutions de saccharose, de chlorure de sodium et de PROP (6-npropylthiouracile) ont permis de déterminer la typologie de goûteur/non-goûteur au PROP et le caractère hédonique (plaisant/déplaisant) des différentes concentrations. Les données ont été analysées en fonction de l'âge, du sexe, du groupe ethnique et des conditions écologiques locales. Les variations des appréciations alimentaires traduisent les stratégies de subsistance, avec des différences significatives entre groupes ethniques correspondant aux conditions environnementales et historiques de chacune des populations considérées. Les facteurs sensoriels, et notamment le statut de goûteur/non-gouteur au PROP, n'apparaissent pas comme des marqueurs pertinents des appréciations alimentaires.

Mots-clés : Perception gustative, saccharose, chlorure de sodium, PROP, préférences alimentaires, populations autochtones arctiques.

ABSTRACT

A study of food preferences of the indigenous peoples of Arctic Yakutia was conducted on a population sample consisting of 102 Evens, 52 Yakuts and 22 Chukchis. Responses recorded on analogue scales corresponding to 69 food items have been supplemented by blind tests concerning the perception of solutions of sucrose, sodium chloride, and PROP.

1. CNRS-UPR 2147, Dynamique de l'évolution humaine, 44 rue de l'Amiral Mouchez, 75014 Paris, France, e-mail : jrl@ivry.cnrs.fr

2. CNRS-UMR Éco-Anthropologie et Ethnobiologie, Muséum National d'Histoire Naturelle, 57 rue Cuvier, 75005 Paris, France, e-mail : ppasquet@mnhn.fr 
to determine hedonic responses to salt and to sucrose (sweet likers vs. sweet dislikers) and the PROP status (tasters vs. non-tasters). Data were computed in relation to age, sex, ethnic groups and ecological factors. Subsistence patterns and food preferences appear to be related, and significant ethnic differences in mean preferences mirror the environmental and historical backgrounds of the various populations. Sensory factors, and notably the PROP status, do not appear as significantly determining food preferences.

Key words: Gustatory perception, sucrose, sodium chloride, PROP, food preferences, aboriginal Arctic societies.

\section{INTRODUCTION}

Le comportement alimentaire de l'homme est sous la dépendance de plusieurs facteurs, les uns endogènes tels l'aptitude sensorielle, les affects, l'expérience, les autres exogènes comme l'histoire, la culture, l'écologie (Macbeth 1997). Plus précisément, la variabilité du comportement alimentaire des populations peut s'expliquer par des mécanismes d'adaptation à des environnements particuliers susceptibles de se modifier dans le temps. On a pu montrer ainsi que les variations de la perception gustative - dont le rôle est admis dans le jeu des préférences et des choix alimentaires - sont impliquées dans l'équilibre alimentaire des groupes vivant dans différents environnements contraignants (Hladik et al. 1986 ; Hladick, Pasquet 1999).

Le présent travail s'inscrit dans cette perspective écologique, à partir d'une étude des préférences alimentaires de groupes ethniques qui vivent dans les milieux arctiques particuliers de Sibérie nord-orientale, pour apprécier leur adéquation aux stratégies de subsistance (Chichlo 1998, 1999). L'accent sera mis sur les variations de la perception gustative, notamment de certains composés amers tel le 6-n-propylthiouracile (PROP), génétiquement déterminée (Fox 1932), qui pourraient être des marqueurs des préférences alimentaires (Fisher et al. 1961 ; Pasquet et al. 2002; Simmen et al. 2003).

Ce travail repose sur les données de terrain obtenues au cours d'une mission effectuée en Yakoutie arctique en 2000. Il s'intègre dans un programme de recherche sur les changements anthropobiologiques et socioculturels des sociétés arctiques, et complète l'étude réalisée en 1997 dans la Basse Kolyma en l'étendant à deux villages du district de Moyenne Kolyma de composition ethnique homogène (Malet 1998 ; Malet et al. 1999, 2002 ; Robert-Lamblin 1998, 2001).

\section{ASPECTS DE L'ÉCOLOGIE ET DE L'ANTHROPOLOGIE ALIMENTAIRE DES POPULATIONS ÉTUDIÉES}

Cette étude couvre deux districts de la Yakoutie nord-orientale, la Basse Kolyma (Nijnekolymski oulous) et la Moyenne Kolyma (Srednekolymski oulous). Ces deux régions représentent deux milieux relativement contrastés, la Basse Kolyma étant le domaine de la toundra arctique qui revêt des aspects variés, parmi lesquels un paysage incluant des mélèzes de petite taille, est observé uniquement à la limite sud-est, alors que la Moyenne Kolyma présente davantage un aspect de «petite » taïga où les mélèzes et quelques bouleaux donnent un paysage plus fermé. La plupart des pâturages de la Basse Kolyma sont couverts par la toundra arctique franche tandis que les pâturages de la Moyenne Kolyma se trouvent dans la zone forestière de mélèzes que les géographes russes désignent du terme « taïga moyenne » (Matveev 1989).

Par ses aspects environnementaux, le district de Srednekolymski répond davantage aux pratiques anciennes des peuples migrants des régions méridionales. Son territoire qui couvre le vaste bassin de la Moyenne Kolyma et qui descend jusqu'au $66^{\mathrm{e}}$ parallèle nord franchit ainsi le cercle polaire par le Sud. La région de Srednekolymski, avec une isotherme de juillet à $14^{\circ} \mathrm{C}$, des sols mieux drainés, une couche d'humus généralement plus épaisse, offre des conditions plus favorables à la croissance végétale.

Dans les deux districts étudiés, vivent des populations de différentes ethnies : Évènes, Yakoutes, Tchouktches, que des immigrants russes ont parfois précédées. Il convient de préciser que, parmi les peuples autochtones, seuls les Yakoutes et les Évènes sont représentés dans chacun des districts, alors que les Tchouktches, nomades éleveurs de rennes, sont essentiellement présents dans les parties septentrionales de toundra (Basse Kolyma). La plupart des ancêtres des familles yakoutes et évènes sont venus s'installer dans la Basse Kolyma à partir de la région voisine qui, en raison de son climat un peu plus clément, a subi les conséquences de la pression démographique sur les ressources naturelles de chasse et de pêche.

La population de l'ensemble du district de la Moyenne Kolyma (oulous de Srednekolymsk) est composée, d'après le recensement administratif de l'année 1999, à $78 \%$ de Yakoutes (Sakhas) et à $4 \%$ seulement d'Evènes, auxquels s'ajoutent $0,2 \%$ d'Evenks et $18 \%$ d'autres nationalités allochtones. Loin d'être dispersés et mêlés aux autres ethnies, comme cela est habituel dans l'Arctique sibérien, les Evènes de la Moyenne Kolyma demeurent un groupe homogène.

Yakoutes et Evènes appartiennent à deux traditions différentes : les premiers sont des éleveurs de chevaux et de bovins, les seconds initialement chasseurs à dos de renne, sont devenus, à partir du XIX ${ }^{\mathrm{e}}$ s., des éleveurs de grands troupeaux de rennes, adoptant ainsi le mode de vie des Tchouktches. De la même façon, les Yakoutes d'origine méridionale, lorsqu'ils se sont installés dans la région de Basse Kolyma, sont devenus des pêcheurs comme les premiers habitants de cette zone, les Youkaghirs (Khudjakov 1969 ; Gurvitch 1977 ; Malet 1990).

Ainsi, chacune des deux régions étudiées présente, sur le plan alimentaire, des particularités qui peuvent s'expliquer à la fois par le milieu et par les traditions culturelles. 
Dans la région de Basse Kolyma (Kolymskoïe et Krasnouchka), d'une façon générale, l'alimentation est riche en produits d'origine animale : viande de renne, gibier et poisson. Par exemple, nous avons pu évaluer sur plusieurs jours, par l'observation de la vie dans un campement (Malet et al. 1999), la quantité de viande consommée chez les éleveurs de renne et notre estimation allait de 800 grammes à $1 \mathrm{~kg}$ par jour et par personne adulte.

Dans les habitudes alimentaires des populations actuelles de l'Arctique, le sucre et les produits céréaliers ont été largement adoptés à partir du XIX ${ }^{e}$ S. (Bogoras 1904 ; Serochevski 1993). Il n'en demeure pas moins que la consommation de viande provenant des ressources locales reste primordiale aux yeux des éleveurs de rennes et de leur famille. Ce type d'alimentation fortement carnée s'apparente chez eux à une valeur identitaire.

L'apport de végétaux dans le régime alimentaire des habitants de la toundra sibérienne demeure très restreint, même si durant la saison chaude la collecte des baies et des champignons peut parâtre une occupation importante (Malet 1995). Peu de plantes de la toundra sont encore utilisées de nos jours dans l'alimentation des populations autochtones, à l'exception de la ciboulette sauvage (Allium schoenoprasum) et de l'oseille arctique (Rumex arctica). En dehors des réserves que l'on prépare pour l'hiver, avec les baies et les champignons de l'été et de l'automne, il faut compter sur les très rares et fort coûteux produits d'importation.

Les épices et le sel ont été diversement adoptés. En général, ils font maintenant partie des ingrédients habituellement utilisés dans la cuisine. Pourtant, Wrangel en explorant la région de Nijne Kolymski en 1821 notait : « Les nomades de la Sibérie ont la plus grande répugnance pour le sel et n'en font jamais usage » (Wrangel 1843, $1: 274$ ) ; et ailleurs : «Si l'étranger demande du sel, on lui en sert, mais jamais autrement ; car les gens du pays ont, pour cet assaisonnement, le dégoût le plus prononcé » (ibid., p. 163). De nos jours, les femmes tchouktches les plus âgées insistent encore sur le fait qu'il faut « très peu » de sel dans les préparations alimentaires.

Dans cette région où les contacts interethniques existent depuis plusieurs siècles, la diversité culturelle s'est en partie estompée. Les différences les plus marquées demeurent sans doute entre le groupe des Yakoutes - le plus tardivement arrivé - et les autres populations autochtones.

Dans la région de Moyenne Kolyma, les habitudes alimentaires diffèrent parmi des populations vivant dans un même environnement. Quoiqu'installés à proximité les uns des autres, les Evènes, chasseurs et éleveurs de rennes, et les Yakoutes, éleveurs de bovins et de chevaux, ont en effet développé des pratiques culinaires et des habitudes alimentaires extrêmement diverses. On peut citer, par exemple, les produits laitiers qui constituent la base nutritionnelle essentielle des Yakoutes, mais qui se trouvent totalement absents du régime alimentaire des Evènes.

Sur le plan alimentaire, tout ce qui provient du renne constitue la nourriture la plus recherchée par les Evènes : « depuis la viande jusqu’à la moelle». Dans le renne, parmi les morceaux qui ont en général la préférence des consommateurs, figurent : la langue, le foie, les rognons, le cœur, la cervelle, la moelle, les yeux. Et, sur la carcasse, c'est la partie de la poitrine qui est considérée comme le meilleur morceau. Chaque partie de l'animal est préparée de façon différente, la consommation crue tenant une place importante. "Près du troupeau, on mange la viande de renne saignante, c'est une particularité évène », font remarquer les éleveurs. Mais la réduction des abattages de rennes domestiques, depuis six à sept ans, afin de laisser les troupeaux se reconstituer, pousse la population à se tourner vers la viande d'élan pour la satisfaction de ses importants besoins en protéines animales.

Même s'il fait partie des menus habituels, lé poisson n'est pas un aliment aussi estimé que la chair des mammifères terrestres. «L'élan est moins bon que le renne, et le renne sauvage moins bon que le renne domestique ; quant au poisson, c'est bien lorsque l'on manque de viande ", affirme une informatrice. Les petits gibiers, tels les lièvres, les lagopèdes, les gélinottes et les tétras, ainsi que les oiseaux migrateurs (oies et canards) constituent des aliments d'appoint, de même que les diverses baies sauvages ramassées en été et automne. Quelques particularités alimentaires sont attribuées aux Evènes. Par exemple, les Yakoutes décrivent les Evènes comme des consommateurs de produits fermentés. Plusieurs plats très appréciés des Evènes sont en effet des aliments faisandés. Quant au goût pour la viande de renne saignante (ailleurs consommée soit vraiment crue, soit bien cuite), il est encore considéré comme une spécialité de ces renniculteurs (Robert-Lamblin 2001).

\section{POPULATIONS ÉTUDIÉES ET MÉTHODES}

Les études ont porté sur trois villages et un campement (fig. 1) localisés respectivement en Basse Kolyma : village de Kolymskoïe ( 826 habitants) ${ }^{3}$ incluant le campement de Krasnouchka, et, en Moyenne Kolyma : villages de Bérëzovka (339 habitants) ${ }^{4}$ et de Nalimsk (527 habitants) ${ }^{5}$. Les sujets ont été retenus sur la base du volontariat, parmi les adultes de 16 ans et plus (âge moyen : 37,3 ans $\pm 15,7$ ) dans les groupes ethniques autochtones : Evènes, Tchouktches et Yakoutes.

3. Dont $37 \%$ de Tchouktches, $26 \%$ de Yakoutes, $12 \%$ d'Evènes, $6 \%$ de Youkaghirs et $19 \%$ de Russes et autres.

4. Dont $85 \%$ d'Evènes, $8 \%$ de Yakoutes et $7 \%$ de Russes et autres.

5. Dont $99 \%$ de Yakoutes et $1 \%$ de Russes et autres. 
Les effectifs des personnes ayant participé aux enquêtes et aux tests de gustation sont présentés dans le tableau I. Ils incluent respectivement $61 \%$ de femmes chez les Evènes, $64 \%$ de femmes Tchouktches et $78 \%$ de femmes Yakoutes.

Afin d'obtenir le consentement et la coopération d'un maximum de personnes, notre enquête était précédée d'une rencontre avec la population locale, au cours de laquelle un exposé en russe, la langue véhiculaire, présentait les buts et les modalités de notre recherche. Ayant été bien comprises des habitants, nos investigations ont pu se réaliser dans des conditions satisfaisantes.

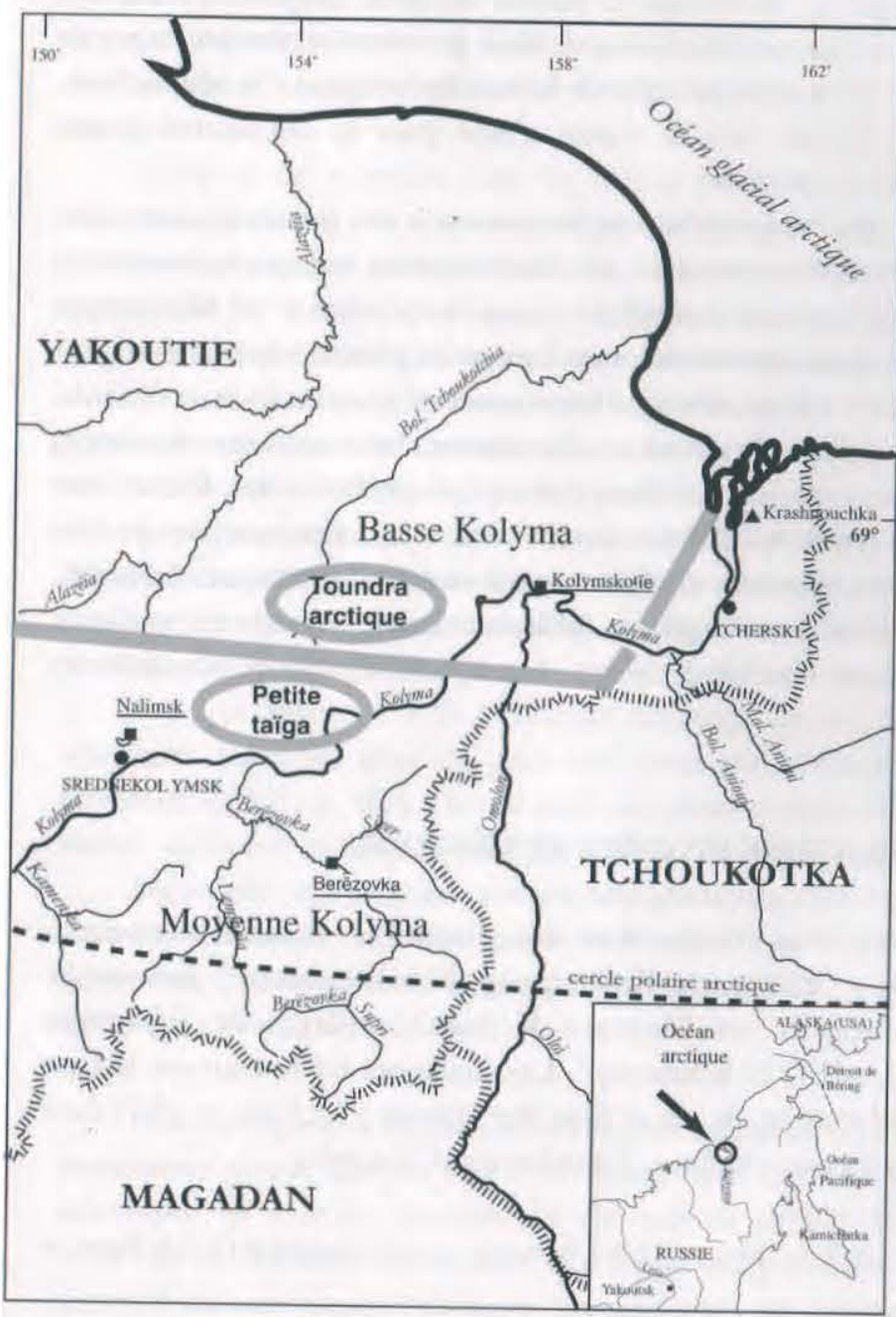

- Ville

- Village étudié
Fig. 1 - Carte de la Yakoutie nord-orientale, indiquant
les lieux étudiés en Basse et Moyenne Kolyma. La limite approximative entre la « petite taïga » et la toundra est indiquée par un trait gris.

Fig. I-Map of north-eastern Yakutia, showing the study sites in Lower and Middle Kolyma. The grey line is an approximate. limit between tundra and taiga. AUTOCHTONES DE YAKOUTIE ARCTIQUE

\begin{tabular}{|l|c|c|c|}
\hline \multirow{2}{*}{$\begin{array}{c}\text { Villages } \\
(n b \text { d'habitants })\end{array}$} & \multicolumn{3}{|c|}{ Ethnies } \\
\cline { 2 - 4 } & Evènes & Yakoutes & Tchouktches \\
\hline Kolymskoïe $(826 h)$ & 12 & 9 & 22 \\
\hline Berëzovka $(339 h)$ & $89(89)$ & $13(13)$ & 0 \\
\hline Nalimsk $(527 h)$ & 1 & $30(30)$ & 0 \\
\hline Total & $102(89)$ & $52(43)$ & 22 \\
\hline
\end{tabular}

Tabl. I - Répartition, en fonction des groupes ethniques et des villages. des 176 participants aux enquêtes sur le goût et les préférences alimentaires (dont, entre parenthèses, le nombre de sujets ayant effectué les tests de perception gustative).

Table I-Distribution of the subjects involved in the study of food preferences (in parentheses, number of the subjects actually tested for gustatory perception). according to ethnic group, in the different villages of Yakutia.
a the

\section{Mesure des appréciations alimentaires}

Les enquêtes ont été menées à l'aide de fiches écrites en russe, contenant chacune 69 items représentatifs de l'alimentation traditionnelle et des usages plus récents. Ces fiches étaient présentées au sujet dans un ordre aléatoire. Les perceptions étaient reportées sur un segment de droite comportant aux extrémités l'apprécation la meilleure et la plus mauvaise des aliments, avec au centre le point de neutralité. Le sujet devait indiquer lui-même par une croix, sur cette ligne, le degré de sa préférence, ou de son dégoût. Le niveau de plaisir/déplaisir évoqué était mesuré par la distance (en $\mathrm{cm}$ ) entre la croix et l'index zéro, en positif pour le plaisir, en négatif pour le déplaisir.

\section{Tests de perception gustative}

Intensités perçues : Des doses de chlorure de sodium, de saccharose et de PROP ayant été préalablement pesées en laboratoire à Paris, étaient, sur le terrain, diluées extemporanément avant chaque test. L'eau potable était utilisée pour les dilutions, incluant 5 solutions de $\mathrm{NaCl}(10 ; 32 ; 100 ; 320$ et $1.000 \mathrm{mM}), 5$ solutions de saccharose $(61 ; 121 ; 242 ; 485$ et $970 \mathrm{mM})$ et 5 solutions de PROP $(0,032 ; 0,1 ; 0,32 ; 1$ et $3,2 \mathrm{mM}$ ). Ces solutions étaient présentées dans un ordre aléatoire au sujet (test en simple aveugle). Entre chaque solution testée, le sujet devait systématiquement se rincer la bouche avec de l'eau.

Après reconnaissance de la qualité présentée : salé, sucré ou amer, l'intensité perçue par le sujet était immédiatement portée par lui-même sur une échelle progressive à 9 points (d'après Likert 1932), codée : 1 = aucune perception, 9 = perception très forte. 
Concernant la perception gustative du PROP et le statut goûteur/non-goûteur (Fox 1932), deux typologies différentes ont été établies :

- En fonction de la sensibilité brute au PROP (d'après Pasquet et al. 2002) en se basant sur une répartition trimodale théorique de la sensibilité pour le PROP. Étaient qualifiés de «non-goûteurs » les sujets ne percevant pas les deux solutions les moins concentrées $(0,032 \mathrm{mM}$ et $0,1 \mathrm{mM})$. Etaient considérés « super-sensibles » les sujets ayant perçu et reconnu la solution la moins concentrée $(0,032 \mathrm{mM})$. Les sujets intermédiaires étaient classés « sensibles ».

- En fonction de la sensibilité au PROP et relativement à l'intensité perçue des solutions de $\mathrm{NaCl}$ (d'après Drewnowski et al. 1997). Étaient qualifiés de « non-goûteurs » les sujets ne percevant pas les deux solutions les moins concentrées $(0,032 \mathrm{mM}$ et $0,1 \mathrm{mM})$ et ayant reconnu les solutions plus concentrées. Étaient considérés « super-goûteurs » les sujets ayant perçu et reconnu la solution de PROP la moins concentrée $(0,032 \mathrm{mM})$ et dont la moyenne des rapports des notes des intensités perçues des 5 solutions de PROP aux intensités perçues des 5 solutions de $\mathrm{NaCl}[(p l / n]+$ $p 2 / n 2+p 3 / n 3+p 4 / n 4+p 5 / n 5) / 5]$ était supérieur à 1,7 . Les sujets intermédiaires étaient classés « goûteurs ».

Tests hédoniques : Le caractère plaisant ou déplaisant de chacune des solutions précédemment décrites était reporté par le sujet sur une échelle verticale dégressive de 9 points également, codée de $: 1=$ extrêmement désagréable à : $9=$ extrêmement agréable.

Conformément à la typologie observée par Thompson et al. (1977), les sujets étaient classés en fonction de la cinétique des notes hédoniques accordées aux 5 solutions de concentration croissante de saccharose. Étaient classés « amateurs de sucre » (sweet likers) les sujets dont la note augmente avec la concentration ou reste constante à un niveau élevé. Étaient classés « non-amateurs de sucre » (sweet dislikers) les sujets dont la note hédonique augmente d'abord, puis diminue rapidement en fonction de la concentration. Les sujets ayant des cinétiques de notes différentes étaient classés « autres ».

\section{Analyse statistique}

L'analyse statistique des données (tests de comparaison de moyennes, analyse de la variance et de la covariance, analyse en composantes principales, régression multiple) a été réalisée à l'aide du logiciel Statistica, version 6.0 (Statsoft, Inc., Tulsa, OK, USA).

\section{RÉSULTATS}

\section{Appréciations des items alimentaires}

Les résultats des appréciations alimentaires sont présentés sur le tableau II où les items sont classés par ordre de préférence en fonction des deux milieux (Basse Kolyma et Moyenne Kolyma) d'une part, et, d'autre part, au sein de chacune des trois ethnies étudiées (Evènes, Yakoutes et Tchouktches).

On note globalement que, dans les deux régions étudiées, les deux items les plus appréciés sont, d'une part la viande de renne, d'autre part les baies de cueillette. Toutefois le renne n'est pas classé au sommet de la liste par les Yakoutes. Il est surprenant par ailleurs que les boissons alcoolisées reçoivent l'appréciation la plus basse dans le contexte de cette étude qui n'était pas marquée de contingences socio-politiques ou religieuses.

Des différences significatives sont apparues dans les appréciations des aliments par les différents groupes ethniques, en particulier en ce qui concerne l'alimentation traditionnelle, comme les viandes d'élan et de cheval, ainsi que le youkola (poisson séché sans sel) ou le koumyss (lait de jument fermenté).

Une analyse factorielle en composantes principales, portant sur l'ensemble de l'échantillon étudié (176 sujets) a été réalisée sur ces appréciations alimentaires (fig. 2).

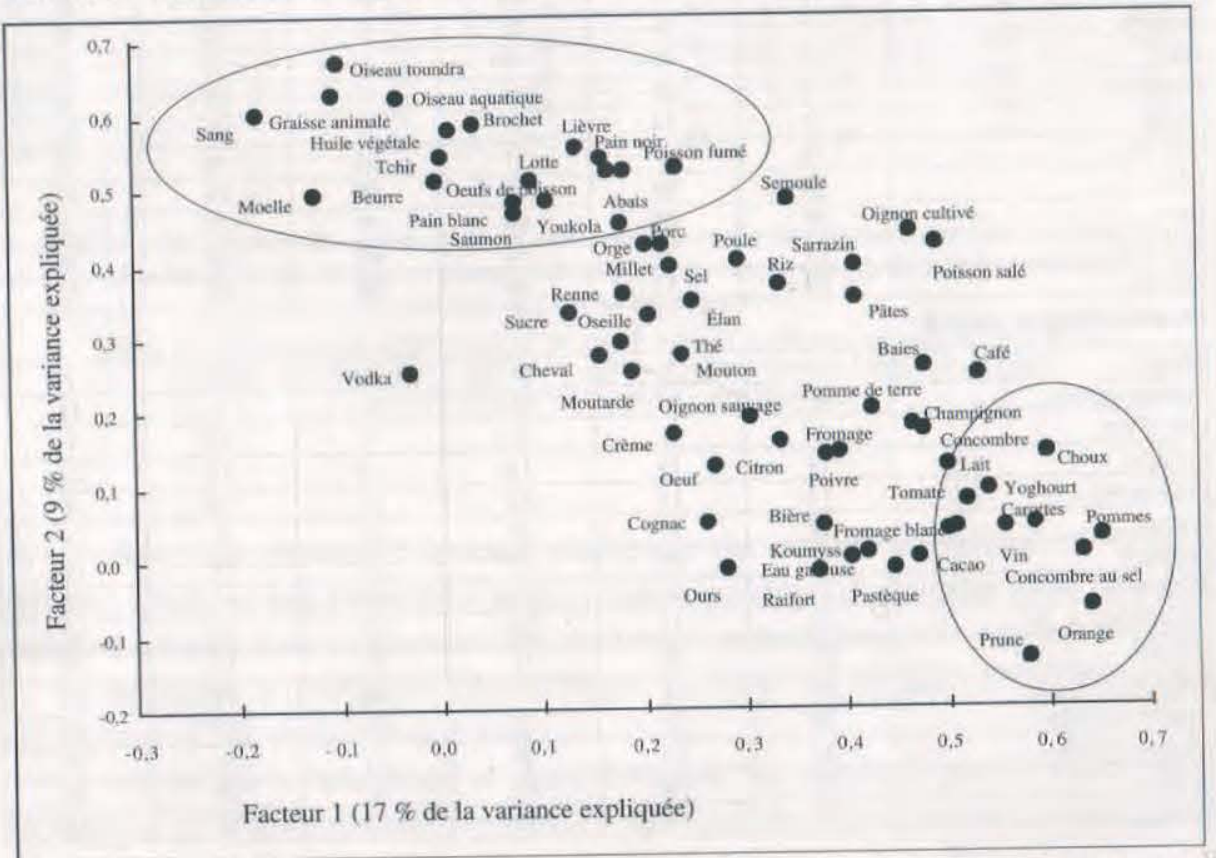

Fig. 2 - Analyse en composantes principales (ACP) des appréciations des différents item alimentaires. Les deux ellipses mettent en valeur respectivement les items qui caractérisent chacun des deux facteurs : qualitatif $(F 1)$ et quantitatif $(F 2)$.

Fig. 2 - Principal component analysis (PCA) of the rates of preferences for various food item. Two major factors are highlighted by the ellipses including respectiv 


\begin{tabular}{|c|c|c|c|c|c|c|}
\hline & Total & $\begin{array}{c}\text { Basse } \\
\text { Koiyma }\end{array}$ & $\begin{array}{l}\text { Moyenne } \\
\text { Kolyma }\end{array}$ & Evènes & Tchouktches & Yakoutes \\
\hline \multicolumn{7}{|c|}{ Produits d'origine animale } \\
\hline Renne & 1 & 2 & 1 & 1 & 1 & 14 \\
\hline Crème & 3 & 13 & 6 & 4 & 6 & 2 \\
\hline Tchir & 5 & 4 & 3 & 5 & 7 & 1 \\
\hline Oeuf & 7 & 17 & 11 & 7 & 2 & 21 \\
\hline Beurre & 9 & 15 & 10 & 9 & 9 & 8 \\
\hline Youkola & 11 & 3 & 7 & 11 & 22 & 12 \\
\hline Abats & 12 & 5 & 15 & 12 & 15 & 31 \\
\hline Lait & 16 & 34 & 23 & 16 & 11 & 7 \\
\hline Moelle & 17 & 11 & 19 & 17 & 18 & 42 \\
\hline Poisson fumé & 19 & 10 & 12 & 19 & 33 & 15 \\
\hline Poisson salé & 20 & 20 & . 21 & 20 & 28 & 6 \\
\hline Oiseau toundra & 24 & 8 & 13 & 24 & 35 & 13 \\
\hline Poule & 25 & 22 & 18 & 25 & 26 & 38 \\
\hline Oeufs de poisson & 27 & 24 & 20 & 27 & 30 & 23 \\
\hline Fromage blanc & 28 & 25 & 32 & 28 & 24 & 37 \\
\hline Élan & 29 & 45 & 44 & 29 & 21 & 27 \\
\hline Oiseau aquatique & 34 & 16 & 22 & 34 & 38 & 19 \\
\hline Mouton & 36 & 36 & 27 & 36 & 36 & 4 \\
\hline Fromage & 37 & 47 & 46 & 37 & 32 & 45 \\
\hline \begin{tabular}{|l} 
Yoghourt \\
\end{tabular} & 39 & 42 & 34 & 39 & 37 & 20 \\
\hline Sang & 41 & 18 & 26 & 41 & 44 & 63 \\
\hline Graisse animale & 43 & 41 & 45 & 43 & 40 & 46 \\
\hline Saumon & 47 & 28 & 39 & 47 & 52 & 58 \\
\hline Porc & 50 & 44 & 40 & 50 & 53 & 43 \\
\hline Koumyss & 52 & 62 & 59 & 52 & 55 & 28 \\
\hline Cacao & 55 & 54 & 52 & 55 & 51 & 60 \\
\hline Lièvre & 56 & 61 & 57 & 56 & 56 & 40 \\
\hline Lotte & 58 & 58 & 60 & 58 & 61 & 41 \\
\hline Ours & 59 & 69 & 66 & 59 & 57 & 55 \\
\hline Brochet & 62 & 50 & 54 & 62 & 62 & 56 \\
\hline Cheval & 64 & 51 & 49 & 64 & 67 & 59 \\
\hline \multicolumn{7}{|c|}{ Produits d'origine végétale } \\
\hline Baies & 2 & 1 & 2 & 2 & 5 & 3 \\
\hline Pomme de terre & 6 & 12 & 9 & 6 & 3 & 11 \\
\hline Pain blanc & 8 & 6 & 8 & 8 & 10 & 9 \\
\hline Pommes & 13 & 19 & 14 & 13 & 13 & 22 \\
\hline Concombre & 14 & 14 & 16 & 14 & 14 & 30 \\
\hline \begin{tabular}{|l} 
Orange \\
\end{tabular} & 15 & 31 & 24 & 15 & 8 & 24 \\
\hline Pastèque & 18 & 23 & 25 & 18 & 19 & 18 \\
\hline Tomate & 21 & 26 & 28 & 21 & 17 & 32 \\
\hline Concombre au sel & 22 & 32 & 29 & 22 & 20 & 17 \\
\hline \begin{tabular}{|l} 
Choux \\
\end{tabular} & 23 & 38 & 31 & 23 & 16 & 25 \\
\hline Riz & 26 & 21 & 17 & 26 & 29 & 34 \\
\hline \begin{tabular}{|l|} 
Oignon sauvage \\
\end{tabular} & 30 & 33 & 37 & 30 & 23 & 50 \\
\hline Oignon cultivé & 31 & 27 & 30 & 31 & 31 & 29 \\
\hline \begin{tabular}{|l|} 
Prune \\
\end{tabular} & 32 & 39 & 42 & 32 & 25 & 39 \\
\hline Pâtes & 35 & 37 & 38 & 35 & 27 & 51 \\
\hline
\end{tabular}

\begin{tabular}{|c|c|c|c|c|c|c|}
\hline & , Total & $\begin{array}{l}\text { Basse } \\
\text { Kolyma }\end{array}$ & $\begin{array}{c}\text { Moyenne } \\
\text { Kolyma }\end{array}$ & Evènes & Tchouktches & Yakoutes \\
\hline \multicolumn{7}{|c|}{ Produits d'origine végétale } \\
\hline Champignon & 38 & 40 & 33 & 38 & 39 & 16 \\
\hline Huile végétale & 40 & 30 & 36 & 40 & 43 & 33 \\
\hline Semoule & 42 & 35 & 41 & 42 & 41 & 47 \\
\hline Pain noir & 44 & 49 & 48 & 44 & 46 & 36 \\
\hline Carottes & 45 & 48 & 50 & 45 & 45 & 53 \\
\hline Sarrazin & 49 & 53 & 51 & 49 & 47 & 44 \\
\hline Betterave & 51 & 57 & 55 & 51 & 49 & 35 \\
\hline Citron & 60 & 59 & 58 & 60 & 60 & 54 \\
\hline Oseille & 61 & 56 & 62 & 61 & 63 & 48 \\
\hline Millet & 66 & 67 & 65 & 66 & 65 & 65 \\
\hline Orge & 67 & 64 & 63 & 67 & 66 & 68 \\
\hline \multicolumn{7}{|l|}{ Condiments } \\
\hline Sucre & 10 & 9 & 4 & 10 & 12 & 10 \\
\hline Sel & 46 & 52 & 47 & 46 & 42 & 52 \\
\hline Moutarde & 53 & 43 & 43 & 53 & 58 & 49 \\
\hline Poivre & 54 & 55 & 56 & 54 & 50 & 61 \\
\hline Raifort & 68 & 68 & 64 & 68 & 68 & 64 \\
\hline \multicolumn{7}{|l|}{ Boissons } \\
\hline Thé & 4 & 7 & 5 & 3 & 4 & 5 \\
\hline Café & 33 & 29 & 35 & 33 & 34 & 26 \\
\hline Eau gazeuse & 48 & 46 & 53 & 48 & 48 & 57 \\
\hline Vin & 57 & 60 & 61 & 57 & 54 & 62 \\
\hline Bière & 63 & 65 & 67 & 63 & 59 & 67 \\
\hline Cognac & 65 & 66 & 69 & 65 & 64 & 66 \\
\hline Vodka & 69 & 63 & 68 & 69 & 69 & 69 \\
\hline
\end{tabular}

Tabl. II - Hiérarchie des appréciations alimentaires (rang des notes moyennes) en fonction du milieu et du groupe ethnique. Les chiffres en caractères gras correspondent à une appréciation négative.

Table II-Hierarchical distribution of the mean rates of preference of various food items, according to environment, and in the different ethnic groups. Data in bold types are for food items negatively appreciated.

Celle-ci fait ressortir 2 facteurs principaux qui expliquent quelque $26 \%$ de la variance totale, et qui permettent, après rotation varimax, d'identifier deux nouvelles variables latentes dans l'expression des préférences alimentaires :

- un facteur « qualitatif » caractérisé par les fruits, les légumes et les condiments, qui explique $17 \%$ de la variance totale.

- un facteur « quantitatif », caractérisé par les aliments gras à haute valeur énergétique (d'origine animale et végétale), qui explique $9 \%$ de la variance totale. 
La structure factorielle mise au jour témoigne, pour ces groupes humains, d'une expression des appréciations alimentaires plutôt en termes de « préférences nutritionnelles » qui doivent être mises en relation, probablement, avec des conditions de vie actuelles (ou passées) en milieu nutritionnellement très contraignant.

Une analyse en fonction du sexe a fait également apparaître des appréciations significativement plus marquées chez les femmes, concernant les fruits et les légumes ( $\mathrm{p}<0,01$ pour betterave, pastèque, pomme, chou), alors que pour les hommes, certains aliments traditionnels comme la graisse de renne ( $p<0,01)$, ainsi que les boissons alcoolisées $(\mathrm{p}<0,01)$ sont davantage appréciés.

Les appréciations sont égalemėnt corrélées avec l'âge des sujets : les plus jeunes apprécient davantage les légumes et les fruits. En revanche, les plus âgés préfèrent les nourritures endogènes comme le gibier ou le sang de renne, ce dont rend compte la corrélation observée d'une part entre l'âge et le score factoriel « qualitatif » $(\mathrm{r}=-0,28$, $\mathrm{p}<0,01)$ et, d'autre part, le score factoriel « quantitatif » $(\mathrm{r}=0,46, \mathrm{p}<0,01)$.

Indépendamment de l'âge et du sexe, il n'y a pas de différences significatives en fonction de la région d'appartenance des sujets (Basse ou Moyenne Kolyma). En revanche, des différences apparaissent entre les différents groupe ethniques, concernant les scores factoriels qualitatifs $(p<0,01)$, quantitatifs $(p<0,05)$ et le niveau moyen d'appréciation (moyenne des notes) des différents items de la liste alimentaire après ajustement par le sexe, l'âge et la région d'appartenance (fig. 3). Les Tchouktches ont un niveau plus élevé d'appréciation des aliments traditionnels carnés et riches en énergie et une moindre acceptation des items relevant du qualitatif (fruits et légumes). D'une manière générale, les Yakoutes acceptent davantage que les autres groupes la liste alimentaire présentée. Toutefois, on n'observe aucune différence entre les différents groupes concernant le nombre d'aliments de la liste inconnus ou jamais consommés.

\section{Tests sensoriels}

La détermination des différentes catégories de goûteurs selon la sensibilité brute au PROP a montré dans l'échantillon, pour un effectif de 132 sujets : 50 super-sensibles (soit $38 \%$ ), 45 sensibles (soit $34 \%$ ) et 37 non-goûteurs (soit $28 \%$ ). Relativement à l'intensité perçue des solutions de $\mathrm{NaCl}$, on obtient une proportion de super-goûteurs de $23,5 \%, 48,5 \%$ de goûteurs et $28,0 \%$ de non-goûteurs.

Si l'on rapproche le statut de goûteur au PROP et les valeurs hédoniques accordées aux solutions des différents produits testés, on observe un lien entre la typologie des classes super-goûteur, goûteur et non-goûteur et le degré de rejet des solutions amères $(\mathrm{F}(2,117)=5,8, \mathrm{p}=0,003)$. Le rejet est le plus important dans le groupe des supergoûteurs. À l'inverse, les non-goûteurs sont ceux qui rejettent le moins les solutions
$\mathrm{F}(2,98)=3,2095 ; \mathrm{p}=0,04465$

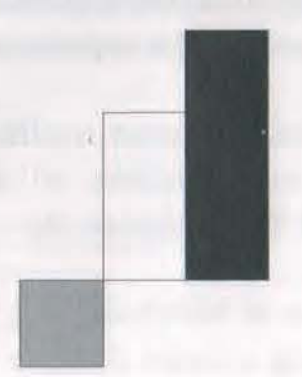

0,80

-

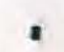

0,00

$-0,10$

$-0,20$

$-0,30$

Score factoriel quantitatif

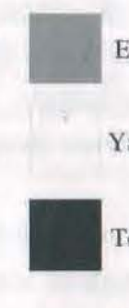

Evènes

Yakoutes

Tchouktches

$30 \quad \mathrm{~F}(2,98)=3,5: \mathrm{P}=0,03$

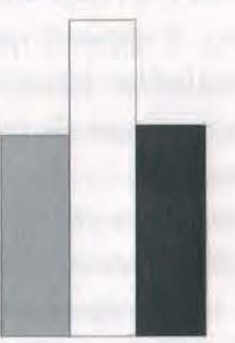

Acceptation moyenne des aliments de la liste
0,60

0,40

0,20

0,00

$-0.20$

$-0,60$

$F(2,98)=4,99 ; p=0,008$

Score factoriel qualitatif

NS

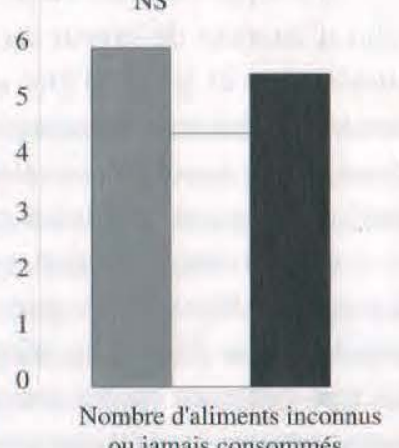

Nombre d'aliments inconnus
$-$

$\bullet$ ig. 3 - Moyennes ajustées (par le sexe, l'âge et la région d'appartenance) des scores factoriels alimentaires qualitatifs et quantitatifs, du niveau moyen d'appréciation de la liste d'aliments et du nombre d'aliments inconnus ou jamais consommés

chez les Evenes, les Yakoutes et les Tchouktches.
Fig. 3-Averages of factorial scores (adjusted according to sex, age, and location) concerning respectively the rate of appreciation of the food items on the list (qualitative vs. quantitative), acceptance, and the number of unknown or never-eaten food items on the list. for the Evens, the Yakuts and the Chukchis. 
amères. Par contre, nous n'avons pas trouvé de relation significative entre le statut de goûteur au PROP et la valeur hédonique accordée aux solutions sucrées.

Il existe une répartition différentielle des deux sexes, dans les statuts de goûteur au PROP, quelle que soit la classification utilisée, avec une proportion plus importante de femmes parmi les sujets les plus sensibles $(p<0,05)$. En revanche, il n'apparaît pas de relation avec l'âge des sujets.

Enfin, le statut de goûteur au PROP a présenté, dans une proportion significative ( $\mathrm{p}<0,01$ ), un plus grand nombre de sujets sensibles (super-sensibles ou supergouteụrs) parmi les Evènes $(31 \%)$ que chez les Yakoutes (12\%), en dépit du plus grand nombre de sujets féminins dans ce dernier échantillon.

Sur les 132 personnes testées, on a relevé : 98 amateurs de sucre (soit $74,2 \%$ de « sweet likers »), 26 non-amateurs de sucre (soit 19,7\% de « sweet dislikers ») et 8 indifférents (soit $6,1 \%$ ). On remarque la forte proportion d'amateurs de sucre : $74 \%$, alors qu'on en trouve rarement plus de $30 \%$ dans les publications spécialisées (Drewnovski et al. 1997). Cette proportion ne varie pas en fonction du sexe ou de l'âge. Par contre, en ce qui concerne l'appartenance ethnique, elle est significativement plus importante chez les Yakoutes que chez les Evènes (respectivement $85 \%$ et $68 \%$; $\left.\chi^{2}=4,3 ; p=0,04\right)$.

Il n'a pas été observé de relation significative entre le statut de goûteur au PROP et celui d'amateur de saveur sucrée. En ce qui concerne la relation entre les appréciations alimentaires et le fait d'être ou non amateur de sucre (sweet liker), il apparaît que ces derniers apprécient davantage certains items à dominante sucrée, telles les baies, ce qui témoigne de la cohérence interne de nos données ; toutefois il n'apparaît pas de relation généralisable aux appréciations des grandes catégories alimentaires.

Concernant la relation entre les facteurs sensoriels et les appréciations alimentaires, il convient de souligner que si les sujets super-sensibles au PROP présentent un plus grand nombre d'items appréciés différemment des autres, pour les sujets super-goûteurs, les non-goûteurs ou les amateurs de sucre, les facteurs qui relèvent du sensoriel ne paraissent pas exercer une grande influence dans les groupes étudiés (aucune différence significative entre les différents groupes observés).

Dans le groupe des Evènes, où nous disposions d'un important corpus de données portant à la fois sur les appréciations alimentaires et la perception gustative, nous avons réalisé les analyses de régression multiple du sexe, de l'âge, et des différentes typologies sensorielles sur les scores factoriels (qualitatif et quantitatif), le niveau moyen d'acceptation et le nombre d'aliments inconnus ou jamais consommés de la liste alimentaire. Bien que des effets dépendants de l'âge et du sexe soient mis en évidence (les sujets féminins apprécient plus les fruits et les légumes, alors que les sujets les plus âgés favorisent plus les items alimentaires quantitatifs), les typologies sensorielles ne semblent en rapport ni avec le niveau moyen des préférences, ni avec le score des facteurs qualitatif et quantitatif des préférences, ni avec le nombre d'items inconnus ou non consommés. Toutefois, nous avons observé une tendance vers un faible score factoriel qualitatif des fruits et légumes (Beta coefficient $=-0,19$, non significatif) chez les sujets super-sensibles au PROP, à âge et sexe constants (tabl. III).

\begin{tabular}{|l|c|c|c|c|}
\hline & Sexe féminin & Ãge & $\begin{array}{c}\text { Sujets super-sensibles } \\
\text { au PROP }\end{array}$ & \% variance expliquée \\
\hline $\begin{array}{l}\text { Score factoriel qualitatif (fruits et } \\
\text { légumes) }\end{array}$ & $0,24^{*}$ & $-0,36^{* *}$ & $-0,19$ & $20 \%$ \\
\hline $\begin{array}{l}\text { Score factoriel quantitatif } \\
\text { (viandelénergic) }\end{array}$ & $-0,30^{* *}$ & $0,8^{* *}$ & 0,04 & $30 \%$ \\
\hline
\end{tabular}

Tabl. III - Analyse de la régression multiple (régression du sexe, de l'âge et du statut de goûteur au PROP) sur les scores factoriels alimentaires qualitatif et quantitatif chez les Evènes, les Yakoutes et les Tchouktches.

Table III -Results of the multiple regression analysis of sex, age, and the PROP status (taster vs, non-taster) on the qualitative and quantitative factors concerning the (aster vs. non-taster) on the qualitative and quantitative factors concerning the
scores of preferences among the Evens, the Yakuts and the Chukchis.

\section{DISCUSSION}

L'analyse des résultats obtenus dans l'étude des préférences alimentaires, pose un certain nombre de questions auxquelles, dans la majorité des cas, la connaissance du milieu environnemental et culturel permet d'apporter des éléments de réponse. C'est ainsi que la prédilection pour une alimentation riche en graisse dans des régions où la température hivernale connaît habituellement des extrêmes de $-40^{\circ}$ à $-50^{\circ} \mathrm{C}$ n'est pas faite pour surprendre. Si l'on ajoute à cela que les populations d'éleveurs sont soumises à des efforts physiques intenses au cours de leur activité quotidienne, on comprend que le recours à des nutriments hautement caloriques réponde à une nécessité biologique (Robert-Lamblin 2003).

Mais au-delà de généralités, certains de nos résultats méritent une réflexion plus approfondie. L'analyse factorielle des préférences alimentaires a fait émerger des données brutes, deux groupes d'âge : les jeunes (au-dessous de 30 ans) et les anciens (plus de $30 \mathrm{ans}$ ), dont les goûts s'opposent radicalement. Ces différences transcendent toutes les autres catégories retenues, que ce soit le sexe, l'ethnie ou le statut goûteur/non-goûteur, au point qu'on est en droit de s'interroger sur la ou les raisons profondes d'une telle opposition. Les causes de ce qui peut apparaître comme un changement dans le comportement alimentaire peuvent être exogènes ou endogènes, 
pour reprendre la dichotomie exposée au début de ce travail. Exogènes, elles pourraient n'être que la manifestation d'une acculturation des jeunes Tchouktches et Evènes au contact de deux populations : les Russes et les Yakoutes qui, les uns et les autres, sont traditionnellement consommateurs de produits laitiers, à la différence des deux premiers. On peut voir aussi l'influence « occidentale » et notamment celle de la société de consommation, omniprésente dans les médias en Yakoutie (vidéofilms, télévision).

À l'origine éleveurs de bovins et de chevaux, les Yakoutes ont conservé diverses habitudes, dont leur goût prononcé pour les produits laitiers, tout en adoptant largement les pratiques alimentaires des autres communautés. Les Tchouktches ont également maintenu des particularités dans leurs préparations culinaires. Cependant, les plus grandes divergences existant de nos jours, dans ce domaine des habitudes alimentaires, viennent essentiellement du type d'activité des individus concernés et de leur lieu d'habitat. La viande de renne et le poisson, tout en demeurant les aliments de base des autochtones sédentarisés dans les villages dont la construction leur fut imposée, ne revêtent pas la même importance lorsqu'il s'agit des éleveurs de rennes ou des pêcheurstrappeurs (Robert-Lamblin 1998).

L'opposition des préférences alimentaires observée entre les sexes, pour être plus nuancée, n'en est pas moins riche de sens, atteignant des dimensions qui dépassent les catégories individuelles, culturelles et environnementales. En d'autres termes, elle conduit à des considérations anthropologiques qui dépassent le cadre de notre article mais qui peuvent éclairer notre interprétation des faits. L'analyse des données rend compte d'un horizon gustatif indéniablement plus riche chez la femme que chez l'homme. En matière de choix alimentaires, celle-ci présentera une palette plus éclectique que celui-là. Ce qui peut, à première vue, paraître paradoxal, c'est que la femme qui fait figure de gardienne de la tradition dans ces sociétés autochtones, au point de l'incarner, va en même temps innover. Le paradoxe n'est que de façade si l'on veut bien admettre que la tradition, c'est-à-dire l'ensemble des idées et des pratiques qui se transmet d'abord au sein de la famille, n'est pas un tout immuable, mais en constante évolution, chaque génération l'enrichissant d'apports divers.

Les préférences rapportées à l'appartenance ethnique et introduisant done un facteur culturel nous ont conduits à la notion d'aliment de substitution. La préférence marquée pour un poisson chez des éleveurs de tradition carnivore comme les Yakoutes avait de quoi surprendre.

De la même façon, la crème occupe globalement le quatrième rang, apparaissant au deuxième rang chez les Yakoutes qui sont de grands consommateurs de laitages (de vache et de jument), au quatrième chez les Evènes, tandis que les Tchouktches ne leur accordent que le treizième, ce qui peut se concevoir chez un peuple de pasteurs qui n'a jamais consommé de manière habituelle le lait de ses rennes.
Le tchir, un poisson d'eau douce (Coregonus nasus), arrive globalement en cinquième position, alors qu'il occupe la première dans le questionnaire des Yakoutes. Le choix de ce poisson particulièrement savoureux peut s'expliquer par ses qualités gustatives, mais il peut être aussi conjoncturel. Il faut savoir que, historiquement parlant, chez les Yakoutes, la pêche était une activité peu considérée et la consommation de poisson moins prisée que celle de viande de bœuf ou de cheval. Tchouktches et Evènes lui reconnaissent une importance à peu près égale. En fait, les Yakoutes, arrivés dans les régions où l'élevage n'était plus possible, ont adopté les activités des peuples environnants et attribuent actuellement à la pêche le même statut prestigieux qu'ils attribuaient auparavant à l'élevage. Ainsi que Khudjakov (1969) l'a observé au XIX ${ }^{e}$ s. « dans la toundra, on s'habitue tellement au poisson que tout autre catégorie alimentaire n'est plus considérée comme de la nourriture ».

À propos de l'absence de relation avec les facteurs sensoriels, les facteurs chimiosensoriels - et notamment, les variations de la perception gustative - doivent être considérés comme le premier maillon de la chaîne explicative des préférences et des choix alimentaires. C'est pourquoi on a avancé l'idée que la sensibilité gustative certains composés amers de la famille des isothiocyanates aromatiques tels que la phénylthiocarbamide (PTC) et le 6-n-propylthiouracile (PROP), qui est génétiquemen déterminée, pouvait être un indicateur des préférences alimentaires (Drewnowski, Rock 1995). Dans une étude récente réalisée en Tunisie (Pasquet et al. 2002), deux d'entre nous ont émis l'hypothèse selon laquelle les sujets qui présentaient une acuité élevée pour le PROP, présenteraient également un comportement alimentaire à tendance néophobique, c'est à dire des préférences accrues pour les items alimentaires ayant une haute valeur hédonique dans la population et des aversions plus accentuées lorsque l'aliment était l'objet de rejet ou de dégoût. Il en résulte une palette alimentaire plus réduite que chez les sujets moins sensibles. Toutefois, la liste des items présentée dans l'étude tunisienne était représentative des aliments les plus consommés, et done, généralement et culturellement appréciés.

Il ressort de nos résultats que les préférences alimentaires manifestées par les populations autochtones de Basse et de Moyenne Kolyma, qui vivent dans de environnements particulièrement contraignants, sont en adéquation étroite avec les stratégies de subsistance et qu'elles s'expriment plutôt en termes de préférences nutritionnelles. Mais il n'a pas été possible de mettre en évidence chez elles l'influence des facteurs sensoriels sur les préférences alimentaires, en dehors de la tendance vers un moindre appréciation des fruits et des légumes chez les sujets les plus sensibles au PROP, ce qui irait dans le sens de certaines conclusions publiées (Drewnowski, Gomez Carneros 2000). 
On peut opposer les sociétés de pêcheurs, incluant une partie des Évènes et des Yakoutes, aux autres sociétés du Grand Nord, en particulier les Tchouktches, chez qui la viande de mammifêre est considérée comme « la nourriture par excellence » (RobertLamblin 2003). C'est l'aliment qui apparaît comme le meilleur parmi tous, tant d'un point de vue gustatif que sanitaire ; et avoir faim, c'est en réalité « avoir faim de viande ", comme dans le cas des peuples des forêts d'Afrique centrale dont le gibier constitue l'aliment idéal (Garine, Pagezy 1990). Se sentir rassasié, c'est avoir consommé de la viande (et la graisse qui l'accompagne indissociablement) ; les autres aliments : poissons, végétaux, et même chair d'oiseau, qui pourtant apportent une variété tout à fait appréciable dans le régime alimentaire, ne constituent en réalité que des en-cas, des coupe-faim précédant un repas véritable ou bien des pis-aller en attendant les jours meilleurs.

Cette conception idéalisée d'une bonne alimentation reposant sur un régime essentiellement carné était traditionnellement transposable également à la vie dans l'au-delà. Les nomades éleveurs de rennes accompagnent, encore de nos jours, leurs rites et leurs célébrations, de sacrifices de rennes domestiques, au cours desquels la consommation collective de la chair de l'animal sacrifié revêt un caractère rituel d'une grande importance symbolique. Cette place primordiale du renne, tant sur le plan nutritionnel que sur le plan symbolique est plus évidente chez les Tchouktches non sédentarisés que nous avons observés dans leur campement. L'analyse des appréciations alimentaires de ce groupe met particulièrement en évidence la relation forte entre le mode de vie et les habitudes alimentaires, que nous avons explorée par cette étude.

\section{Remerciements}

Nous tenons à exprimer nos remerciements à l'Institut Polaire Français Paul Émile Victor (anciennement I.F.R.T.P.) dont le soutien financier a permis la réalisation de ce projet.

\section{BIBLIOGRAPHIE}

BoGoras (W.) 1904 (réédition 1975), The Chukchee: Material Culture, Memoirs of the American Museum of Natural History, The Jesup North Pacific Expedition, VII, 1 .

Chichlo (B.) 1998, Antécédents et contraintes ethniques : problèmes d'identification chez les autochtones de la Basse Kolyma, Boréales, Revue du Centre de Recherches Inter Nordiques $74 / 77$ 7-30.

Chichlo (B.) 1999, Le district de Nijnekolymski : population, renniculture, ethnicité, in Sibérie II, Histoire, Culture, Littérature, Institut d'Études Slaves, p. $475-502$.

DREWNOWSKI (A.), GoMEZ-CARNEROS (C.) 2000 , Bitter taste, phytonutrients, and the consumer: a review, American Journal of Clinical Nutrition 72: 1424-1435.

DREWNOWSKI (A.), HeNDERSON (S.A.), SHORE (A.B.) BARRAT-FORNEL (A.) 1997 Nontasters, tasters, and supertasters of 6-n-propylthiouracil (PROP) and hedonic response to sweet, Physiology and Behavior 62: 649-655.

DREWNOWSKI (A.), ROCK (C.L.) 1995 Influence of genetic taste markers on food acceptance, American Journal of Clinical Nutrition 62: 506-511.

Fisher (R.), GRIFFIN (F.), ENGLAND (S.) GARN (S.M.) 1961, Taste thresholds and food dislikes, Nature 191: 1328

Fox (A.L.) 1932, The relationship between chemical constitution and taste. Proceedings of the National Academy of Sciences 18: 115-120.

Garine (I. de), Pagezy (H.) 1990, Seasonal hunger or "craving for meat"? In
C.M. Hladik, S. Bahuchet, I. de Garine (eds), Food and nutrition in the African rain forest, Unesco, Paris, p. 43-45.

GURVITCH (I.S.) 1977, Kultura severnykh jakutov-olenievodo, Nauka, Moscou.

HLADIK (C.-M), RoBbe (B.), PAgezy (H.) 1986, Sensibilité gustative différentielle des populations Pygmées et non Pygmées de forêt dense, de Soudaniens et d'Eskimos, en rapport avec l'environnement biochimique, Comptes Rendus des Séances de l'Académie des Sciences de Paris, série III, $303: 453-458$.

HLADIK (C.-M), PASQuet (P) 1999 Évolution des comportements alimentaires : adaptations morphologiques et sensorielles, Bulletins et Mémoires de la Société d'Anthropologie de Paris, n.s., 1 , $3-4: 307-332$

KHUDJAKOV (I.A.) 1969, Kratkoje opisanije Verkhojanskogo okruga, Nauka, Léningrad.

LIKERT (R.) 1932, A technique for the measurement of attitudes, Archives of Psychology 140.

MAсветH (H.) (éd.) 1997, Food Preferences and Taste, Continuity and Change, Berghahn Books, Oxford/New York.

Malet (C.) 1990, Les peuples du Nord aujourd'hui, Boréales, Revue du Centre de Recherches Inter Nordiques 40/45 : 1-384.

MALET (C.) 1995, Notes sur l'environnement de la Basse Kolyma avec un inventaire des mammifères, des oiseaux et des baies qu'on peut y observer, Boréales, Revue du Centre de Recherches Inter Nordiques $62 / 65: 159-172$.

MaLET (C.) 1998, Réflexions sur la santé des peuples de Yakoutie : approche 
anthropologique et médicale, Boréales, Revue du Centre de Recherches Inter Nordiques $74 / 77: 66-110$.

Malet (C.), Chichlo (B.), Robert-Lamblin J.) IACONelli (S.), PASQuet (P.), HLADIK (C.-M.) 1999, La perception gustative des populations autochtones de Yakoutie arctique (district de Basse Kolyma) en fonction du régime alimentaire, comparée à celle de populations d'Europe, d'Afrique et du Groenland, Bulletins et Mémoires de la Société d'Anthropologie de Paris, n.s., $11,3-4: 405-416$.

Malet (C.), Chichlo (B.), Robert-Lamblin (J.) 2002, Mission anthropologique en Yakoutie arctique, Districts de Moyenne et de Basse Kolyma, Publication de I'Institut Polaire Français Paul-Emile Victor, Rapport d'activité 2001-2002, p. 79-88.

MATVEev (I.A.) (ed.), 1989, Atlas sel'skogo khozjastva Jakutskoj ASSP, Moscou.

Pasquet (P.), Oberti (B.), El ATI (J.), HLADIK (C.-M.) 2002, Relationships between threshold-based PROP sensitivity and food preferences of Tunisians, Appetite 39: 167-173.

RoBERT-LAMBLIN (J.) 1998, Alimentation et pratiques médicales traditionnelles des populations de la Basse Kolyma (Yakoutie), Boréales, Revue du Centre de Recherches Inter Nordiques 74-77 : 31-65.
ROBERT-LAMBLIN (J.) 2001, Berëzovka : un " isolat " évène en Yakoutie nordorientale, Boréales, Revue du Centre de Recherches Inter Nordique 82/85 : 57-88.

ROBERT-LAMBLIN (J.) (sous presse, 2003), Meat: a food above all others for Arctic Peoples, in A. Hubert (ed.), Meat: Environment, Diet and Health, Berghahn Books, Oxford/New York.

SEROCHEVSKI (V.L.) 1993, Jakuty, Opyt etnografitcheskogo issledovanija, ROSSPEN, Moscou.

Simmen (B.), Pasquet (P.), HLAdIK (C.-M. (sous presse, 2003), Methods for assessing taste abilities and hedonic responses in human and non-human primates, in H. Macbeth, J. McClancy (eds), Research methods for food-related anthropology, Berghahn Books, Oxford.

THOMPSON (D.A.), MosKowITZ (H.R.), Campbell (R.G.) 1977, Taste and olfaction in human obesity, Physiology and behavior 19: 335-337.

WRANGel (Baron F. de) 1843, Le Nord de la Sibérie, Voyage parmi les peuplades de la Russie Asiatique et dans la mer Glaciale, Librairie d'Amyot, Paris, 2 tomes. 